\title{
UNCERTAINTY IN MASS DETERMINATION OF GALAXY CLUSTERS DUE TO THE BULK MOTION OF INTRACLUSTER MEDIUM
}

\author{
M. TAKIZAWA AND S. MINESHIGE \\ Department of Astronomy, Faculty of Science, Kyoto University, \\ Sakyo-ku, Kyoto 606-01,JAPAN
}

\section{Introduction}

Determination of the mass of cluster of galaxies (CG) is very important mainly because it relates to determination of cosmological density parameter $\left(\Omega_{0}\right)$.

However, mass obtained from gravitational lensing tends to be larger than that from X-ray observation with the assumption of hydrostatic equilibrium (HSE) in some CGs (Miralda-Escudeé \& Babul 1995, Schindler et al. 1997). We suggest one of the reason for this discrepancy is that the assumption of HSE is inaccurate because bulk motion of ICM is left. We, therefore, performed the simulation of spherical CG consisting of dark matter and gas.

\section{Methods of Analysis}

We use the model cluster in Takizawa \& Mineshige (1997). Cosmological model is Einestain de Sitter $\left(\Omega_{0}=1, \Lambda_{0}=0\right)$. Spherically symmetric is assumed.

We derive two kind of the estimated mass from the model cluster. One is the mass derived from the density profile and the temperature profile, which we call $M_{\text {est,1. }}$. The other is the that from the density profile and the emissivity weighted temperature, which we call $M_{\text {est,2 }}$. Therefore,

$$
M(r)_{\mathrm{est}, 1}=-\frac{k T(r) r}{G \mu m_{\mathrm{p}}}\left[\frac{d \ln n}{d \ln r}+\frac{d \ln T}{d \ln r}\right]
$$




$$
M(r)_{\mathrm{est}, 2}=-\frac{k r}{G \mu m_{\mathrm{p}}} T_{\mathrm{ew}} \frac{d \ln n}{d \ln r} .
$$

We will compare $M_{\mathrm{est}, 1}$ and $M_{\mathrm{est}, 2}$ to the true mass, $M$.

\section{Results and Discussions}

Figure 1 shows the radial profiles at $z=0$ of $M_{\mathrm{est}, 1} / M$ (solid line) and $M_{\text {est }, 2} / M$ (dotted line), respectively.

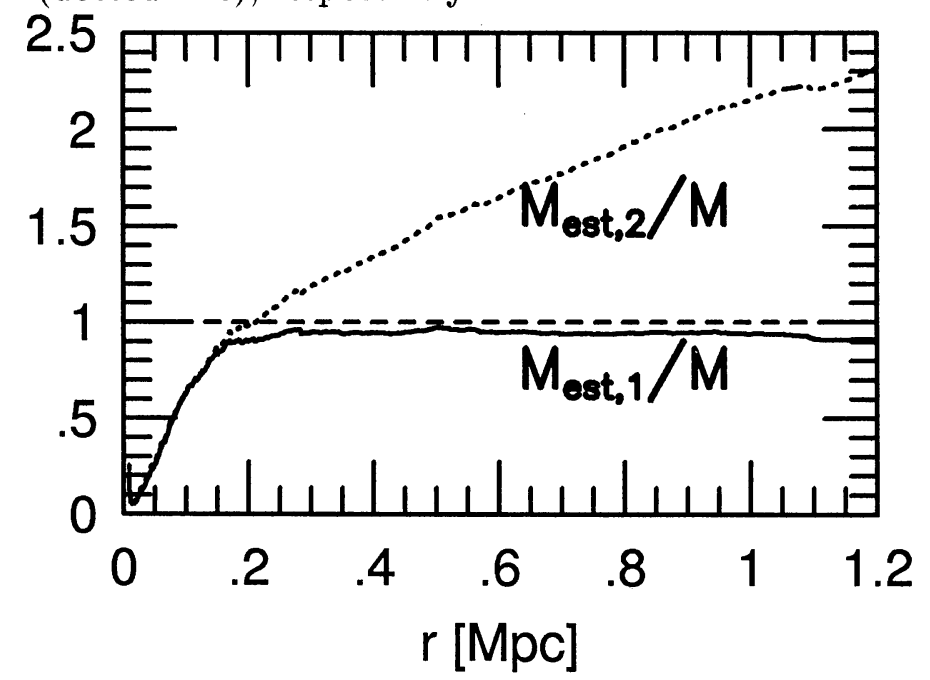

Figure 1. Radial profiles of $M_{\mathrm{est}, 1} / M$ (solid line) and $M_{\mathrm{est}, 2} / M$ (dotted line), respectively.

$M_{\text {est, } 1} / M$ is about 0.95 except in the central region. Therefore the effect of the deviation from HSE is not important. On the other hand, the behavior of $M_{\text {est,2 }} / M$ is rather different, which is monotonically increasing outwards. This is mainly due to the temperature gradient. Therefore, in the case of spherical symmetry, the effect of bulk motion of ICM is not very importance. Note that non isothermality of ICM can have great influence.

According to our results, the observational discrepancy cannot be explained solely due to the effect of bulk motion of ICM. The deviation from spherical symmetry (substructure, projection effect) and uncertainty of modeling of gravitational lensing should be considered in more detail.

\section{References}

Miralda-Escudeé, J. \& Babul, A. 1995, ApJ, 449, 18

Schindler, S., Hattori, M., Neumann, D. M. \& Böhringer, H. 1997, A\&Ap, 317, 646

Takizawa, M. \& Mineshige, S., 1998 accepted for publication in ApJ, astro-ph/9702047 